\title{
Fixed drug eruption associated with fixed combination of fluoroquinolone-nitroimidazole
}

\author{
R. Meenakshi, S. Nitya, S. Kiruthika, M. Shanthi*
}

Department of Pharmacology, Sri Manakula Vinayagar Medical college and Hospital, Pondicherry, India

Received: 22 February 2021

Accepted: 31 March 2021

*Correspondence:

Dr. M. Shanthi,

Email: shanthibabu24@gmail.com

Copyright: (C) the author(s), publisher and licensee Medip Academy. This is an open-access article distributed under the terms of the Creative Commons Attribution Non-Commercial License, which permits unrestricted non-commercial use, distribution, and reproduction in any medium, provided the original work is properly cited.

\begin{abstract}
Fixed drug eruptions (FDE) are cutaneous adverse drug reaction characterized by well demarcated erythematous plaques which on removal of the offending agent resolves with residual hyperpigmentation patches at the site. FDE to nitroimidazoles and fluoroquinolones have nevertheless been infrequently reported. Awareness about the adverse reaction to the fluoroquinolone-nitroimidazole combination drug and also the likelihood of recurrence with same or similar drugs and the possible cross reaction is eminent. Hereby we report one such case of FDE to ciprofloxacin/tinidazole combination.
\end{abstract}

Keywords: FDE, ciprofloxacin, Tinidazole

\section{INTRODUCTION}

Fixed drug eruptions (FDE) are cutaneous adverse drug reaction characterized by well demarcated erythematous plaques which on removal of the offending agent resolves with residual hyperpigmentation patches at the site.

The incidence is often between 20 and 40 years of age. ${ }^{1}$ The lesions often occur at sites such as extremities, lips, genitalia and perianal regions. Antimicrobials are one of the most common drugs implicated to cause FDEs, especially reaction to nitroimidazoles and fluoroquinolones have nevertheless been infrequently reported. ${ }^{1-3}$ Cross-reaction between fluoroquinolones, nitroimidazoles, fluoroquinolone-nitroimidazole combinations have also been previously reported. ${ }^{1,4}$ Awareness about the adverse reaction to the fluoroquinolone-nitroimidazole combination drug and also the likelihood of recurrence with same or similar drugs and the possible cross reaction is eminent.

Hereby we report one such case of FDE to ciprofloxacin/tinidazole combination.

\section{CASE REPORT}

A 39-year-old female patient presented with fever, nausea and vomiting. She was diagnosed as acute gastroenteritis and was prescribed with tablet ciprofloxacin 500 $\mathrm{mg} /$ tinidazole $600 \mathrm{mg}$ fixed dose combination (FDC) twice daily along with tablet paracetamol.

After one day of consuming the medication, patient developed generalized multiple fluid filled eruptions over the upper arms which ruptured spontaneously leaving hyperpigmented patch at the sites on discontinuation of the FDC.

Upon further inquiry, she had no known allergy to paracetamol and also revealed that she had no history of exposure to the FDC of ciprofloxacin and tinidazole combination in the past. On the Naranjo's causality assessment scale, the adverse event was 5 indicating a 'probable' reaction to the FDC. 


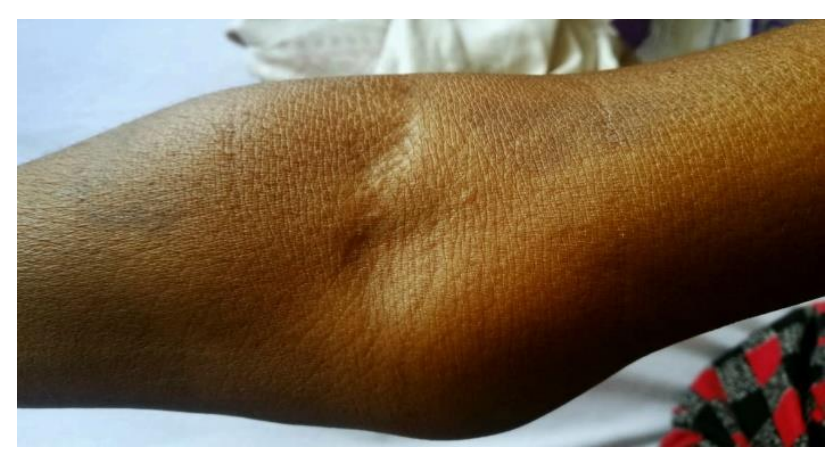

Figure 1: Hyperpigmented patches over the arm.

\section{DISCUSSION}

FDC containing ciprofloxacin a broad-spectrum quinolone antibiotic and tinidazole a nitroimidazole derivative antiprotozoal is widely used in the treatment of diarrhoea in India. ${ }^{2}$ However, these FDC are not a part of WHO essential medicine list. Incidence of FDE, drug-induced anaphylaxis and erythema multiforme and vomiting have been reported with the use of Ciprofloxacin/tinidazole combination. ${ }^{2,3,5}$ Adverse drug reaction to this FDC makes it difficult to determine the possible offending drug due to cross sensitivity. ${ }^{1}$ FDE involves activation of cellmediated cytotoxic response with the offending drug act as a hapten that binds to basal keratinocytes, leading to an inflammatory response. ${ }^{5}$ Though rechallenge with the offending drug is the gold standard for confirmation of FDE, in this case it was not attempted as the patient was not willing for the fear of aggravation of the lesions.

\section{CONCLUSION}

Judicious use of the ciprofloxacin/tinidazole FDC is required in view of cosmetic disfigurement seen with
FDEs. Patients should be counselled on recurrence of FDE with the offending drug and possible cross-reactions of similar medications.

\section{Funding: No funding sources \\ Conflict of interest: None declared \\ Ethical approval: Not required}

\section{REFERENCES}

1. Jhaj R, Chaudhary D, Asati D, Sadasivam B. Fixeddrug Eruptions: What can we Learn from a Case Series? Indian J Dermatol. 2018;63(4):332-7.

2. Jayanthi CR, Chaithra KN, Reddy NS. A profile of adverse drug reactions to antimicrobial agents at a tertiary care hospital. Indian J Pharm Pharmacol 2017;4(1):16-21.

3. Ciprofloxacin/tinidazole. Reactions Weekly 1547. Springer Nature. 2015;91.

4. Mithari HS, Gole PV, Kharkar VD, Mahajan SA. Generalized Bullous Fixed Drug Eruption to Fluconazole; with Cross-Reactivity to Tinidazole. Indian J Dermatol. 2019;64(4):335-7.

5. Sahoo, Himanshu, Panda, Abinash, Rath, Bandana. The unseen story of Tinidazole: Alarm to the drug regulatory bodies. MOJ Clin Med Care Rep. 2015;00007.

6. Shiohara T. Fixed drug eruption: pathogenesis and diagnostic tests. Curr Opin Allergy Clin Immunol. 2009;9(4):316-21.

Cite this article as: Meenakshi R, Nitya S, Kiruthika S, Shanthi M. Fixed drug eruption associated with fixed combination of fluoroquinolonenitroimidazole. Int J Basic Clin Pharmacol 2021;10:576-7. 